\title{
Precise Interception of a Maneuvering Target by a Missile
}

\author{
${ }^{1}$ Mehmet Önder Efe, ${ }^{2}$ Okyay Kaynak and ${ }^{3}$ Bogdan M. Wilamowski \\ ${ }^{1}$ Carnegie Mellon University, Electrical and Computer Engineering Department, Pittsburgh, PA 15213-3890, U.S.A. \\ ${ }^{2}$ Bogazici University, Electrical and Electronic Engineering Department, Bebek, 80815, Istanbul, Turkey \\ ${ }^{3}$ University of Idaho, Graduate Center at Boise, 800 Park Blvd., Boise, U.S.A.
}

\begin{abstract}
This paper presents a new error critic for neurocontrol of highly nonlinear systems. The unavailability of the target outputs of a neural network controller constitutes a difficulty in tuning the adjustable weights and biases and deserves a careful consideration to improve the tracking performance. The method described aims to drive the system into a predefined sliding regime through the use of a novel error measure. The controller is an ordinary feedforward neural network, the tuning strategy is the error backpropagation and the contribution of the paper is the proof of precise tracking by using the proposed error critic in such a standard neurocontrol configuration. The results obtained justify the performance claims of the paper without requiring the analytical details of system under control.
\end{abstract}

\section{INTRODUCTION}

One of the challenges in the realm of control engineering is the design of control strategies or autopilots for missiles. What make the missile control problem a challenge are the existence of nonlinearities in the system dynamics, multivariable structural representation, strong inter-dependencies between the variables involved and the uncertainties stemming from the modeling errors and environmental factors. A closer look to the studies appeared in the recent literature suggests several interesting strategies for the missile control problem. In the first group, the use of Variable Structure Control (VSC) has been emphasized [1$3]$. The second group focuses on the use of computational intelligence, i.e. neural networks, fuzzy inference systems and genetic algorithms, or some hybrid integration of them $[2,4-7]$. The typical problems associated with the presented approaches has been the model dependency for VSC-type controllers, the need for identification for neural and or fuzzy type controllers and the parametric stability (convergence) problem in all intelligence based control schemes. Alternative methods have generally utilized the local linearization of the derived nonlinear model, and the design based on linear dynamics, feedback linearization of the nonlinear model. Apparently the mentioned strategies increase the model dependency and enforce the validation of the linearized dynamics through a series of costly tests.

One idea to overcome the mentioned difficulties is to embed the VSC as an integral part of the learning process. Studies discussing this integration have demonstrated that the Sliding Mode Control (SMC) technique can successfully be used for parameter adaptation in intelligent systems. Some remarkable examples utilize adaptive linear elements [8-9], feedforward neural networks [10-11] and fuzzy inference systems [12-13]. Although it is possible to design a tuning law based on VSC, the plant under control can also be forced to a predefined sliding manifold by sliding mode tuning for sliding mode control. In [14], an example of this has been discussed for the control of an anthropoid robotic manipulator.

In this paper, we demonstrate a precise VSC of a missile. The controller is a standard three-layered feedforward neural network, and the tuning mechanism is the well-known error backpropagation technique. It must be emphasized that the controller and its training scheme are the mostly utilized couple that appeared in the literature particularly during the 1990 s. However, the later studies have reported that the mentioned scheme suffers from the oversensitivity against noise and abruptly changing plant parameters. The prognosis was the presence of sharp valleys on the cost hypersurface, on which the gradients are computed. A detailed discussion of eliminating the adverse effects of gradient techniques with VSC technique has been presented in [12-13]. However, the encountered difficulty was the computational burden. Having this motivation behind what we present next, if the task is the sliding mode control of a missile, the error measure that is to be backpropagated can be changed such that the system enters the desired sliding regime.

This paper is organized as follows: The second section introduces the missile control problem, the third section presents the suggested control error. In the fourth section we present the simulation results and the concluding remarks constitute the last part of the paper.

\section{FORMULATION OF THE CONTROL PROBLEM}

The control problem is to intercept the target by the missile having the kinematics described as in (1)-(3). The missile and the target are assumed to be point masses and the representation below is derived for spherical coordinates. The details concerning the model are given in $[1,14]$.

$$
\begin{gathered}
\ddot{r}-r \dot{\phi}^{2}-r \dot{\theta}^{2} \cos ^{2} \phi=a_{T r}-a_{M r} \\
r \ddot{\theta} \cos \phi+2 \dot{r} \dot{\phi} \cos \phi-2 r \dot{\phi} \dot{\theta} \sin \phi=a_{T \theta}-a_{M \theta} \\
r \ddot{\phi}+2 \dot{r} \dot{\phi}+r \dot{\theta}^{2} \cos \phi \sin \phi=a_{T \phi}-a_{M \phi}
\end{gathered}
$$

In above, $r$ is the distance between the missile and the target, $\theta$ and $\phi$ are the azimuth and pitch angles respectively. Furthermore, $a$ stands for the acceleration with $M$ subscript for the missile and with $T$ subscript for the target; the relevant state variable is the second subscript in the 
acceleration inputs. With these terms, the control objective is to reach the maneuvering target by appropriately altering the acceleration components $a_{M}$.

\section{PROPOSED ERROR CRITIC}

Define $\underline{x}=\left[\begin{array}{lll}r & \theta & \phi\end{array}\right]^{T}$ and $\underline{u}=-\left[\begin{array}{lll}a_{M r} & a_{M \theta} & a_{M \phi}\end{array}\right]^{T}$. The system in (1)-(3) can be rewritten as follows:

$$
\underline{\ddot{x}}=\underline{f}(\underline{x}, \underline{\dot{x}})+b(\underline{x}) \underline{u}
$$

If $\underline{x}_{d}$ is defined to be the vector of desired trajectories, one can describe the tracking error vector as $\underline{e}=\underline{x}-\underline{x}_{d}$ and construct the control signal that derives the system of (4) towards the prescribed sliding regime. The design is based on a two-sided switching mechanism, the argument of which is defined as $\underline{s}_{p}=\mathrm{d} \underline{e} / \mathrm{d} t+\Lambda \underline{e}$ with $\Lambda$ being a positive definite diagonal matrix of appropriate dimensions. The aim is to ensure the negative definiteness of the Lyapunov function $V_{p}=\underline{s}_{p}{ }^{\mathrm{T}} \underline{s}_{p} / 2$. The control sequence can now be formulated as below.

$$
\underline{u}=-b^{-1}(\underline{x})\left(\underline{f}(\underline{x}, \underline{\dot{x}})+\Lambda \underline{\dot{e}}+\Xi \operatorname{sgn}\left(\underline{s}_{p}\right)-\underline{\ddot{x}}_{d}\right)
$$

where, $\Xi$ is a positive definite diagonal matrix. The application of the well-known sliding control law above for the system of (4) enforces the following dynamics, which ensures the reaching to the hyperplane $\underline{s}_{p}=\underline{0}$.

$$
\underline{\dot{s}}_{p}=-\Xi \operatorname{sgn}\left(\underline{s}_{p}\right)
$$

Proposition: If $\underline{s}_{C}$ is defined to be the vector of discrepancies between the target and evaluated values of the control vector, and if the controller parameters are adjusted such that the cost function in (7) is minimized, the tracking error vector is driven towards the switching manifold.

$$
J=\frac{1}{2} \underline{s}_{C}^{T} \underline{s}_{C}
$$

where, $\underline{s}_{C}$ is defined to be the error on the control signal and is computed as given in (8).

$$
\underline{s}_{C}=\underline{\dot{s}}_{p}+\Xi \operatorname{sgn}\left(\underline{s}_{p}\right)
$$

Practically, for second order systems, we have the geometric interpretation shown in Fig. 1. In this figure, the subscript $i$ is for the individual entries of the vector $\underline{x}$. Clearly, for the trajectories like $C_{1}$ and $C_{3}$, the error vector $\left[e_{i}, \mathrm{~d} e_{i} / \mathrm{d} t\right]$ tends to the sliding manifold and due to the dynamics characterized by the locus $s_{p i}=0$, the error and its derivative will be forced towards the origin along with the sliding manifold. For these trajectories, the error caused by the controller are considerably smaller in magnitude than the error for trajectories like $C_{2}$ and $C_{4}$, which have the tendency to get away from the sliding manifold. Therefore, the two terms of the right hand side of (8) will have the same signs and when added to each other, the result will be greater in magnitude than what is observed with convergent trajectories. Our first conclusion from this interpretation is that the error critic in (8) is compatible with the design goals and is a suitable measure for tuning the controller parameters. The second conclusion is on the parametric growth. If the convergent trajectories of Fig. 1 are compared with divergent ones, one would directly infer that the error in (8) inactivates the tuning mechanism in the vicinity of the sliding manifold, however the conventional tuning schemes gradually update the controller parameters until the origin is reached.

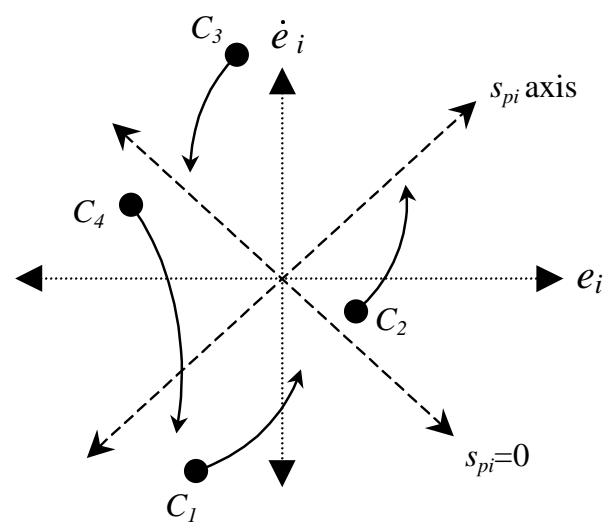

Fig. 1. Graphical interpretation of the suggested error critic

At this point, we dwell on what is questionable with this error measure. The obvious difficulty is the computation of the time derivative of $\underline{s}_{p}$. Our prior tests have proved that an approximate numeric differentiation works even with the noisy observations. Introducing a stable linear filter with numerator of order one in Laplace domain can suitably provide the information needed. The reason why we do not need the exact value of the derivative stems from the fact that the desired behavior is not unique. If a trajectory starting from an arbitrary initial point in the space shown in Fig. 1 tends to the sliding manifold then it is one of the desired trajectories, however, the selection of $\Xi$ uniquely determines the way of approaching the sliding manifold. The information loss due to the derivative computation can be interpreted as a slight modification of the reaching dynamics of (6). The second question is on the selection of the diagonal positive definite matrix $\Xi$. If the entries increase in magnitude, the reaching phase of the control strategy produce large controls in magnitude and several hittings occur, however, the values close to zero result in slow reaching to the sliding manifold with relatively less number of hittings. The designer has to decide on what he/she pursues together with the physical reality regarding the plant under control. For example, for a cargo ship steering example, enforcing the convergence to a desired behavior in 
a few seconds would require unrealistically large-magnitude control activity, while for a direct drive manipulator the response could reasonably be fast to fulfill the imposed task. Lastly, the infinite switching frequency of ideal sliding mode should be addressed. Clearly from (6), one should notice that the enforced behavior ultimately converges to a practically impossible phenomenon. Since the right hand side of (6) is discontinuous in the vicinity of the origin, the near origin activity is an oscillation ideally at infinite frequency, called chattering in the terminology of sliding control. One approach to eliminate the adverse effects of chattering is to introduce a boundary layer by replacing the discontinuous sign function with a smooth approximate such as the one given below.

$$
\operatorname{sgn}(\alpha) \cong \frac{\alpha}{|\alpha|+\delta}
$$

where $\delta>0$ is the parameter determining the accuracy of the approximation. In what follows, we focus on the control of missile by a simple neural network utilizing the error critic in (8) for parameter adjustment.

\section{SIMULATION RESULTS}

\section{A. A Feedforward Neural Network Controller}

Due to the powerful mapping capabilities, artificial neural networks are preferred in many applications requiring precision in nonlinear mapping (control, identification), fault tolerance (VLSI, microelectronics), generalization (image processing and pattern recognition). Various architectures with a considerable amount of diversity in tuning schemes result in the fact of algorithmic and architectural integrity, which is the underlying feeling of the designers utilizing neural systems.

In Fig. 2, a three-layered feedforward neural network is illustrated with $m$ neurons in the input layer and $n$ neurons in the output layer.

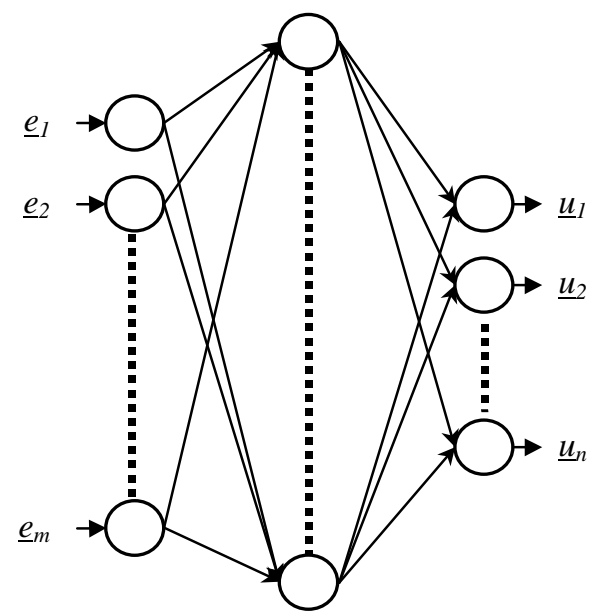

Fig. 2. A three-layered feedforward neural network structure with $m$ inputs and $n$ outputs.
Since the plant under control has six state variables, the number of inputs is six, and the number of outputs is three, i.e. $m=6, n=3$. In the simulations, we utilize $\Psi(\alpha)=\tanh (\alpha)$ as the neuronal activation functions for hidden layer neurons, and $\Psi(\alpha)=\alpha$ for the output layer neurons. Having this in mind, the I/O relationship of the depicted structure can be described as follows:

$$
\underline{u}=W_{R}^{T} \underline{\Psi}\left(W_{L}^{T} \underline{e}-B_{L}\right)-B_{R}
$$

where $W_{R}$ and $W_{L}$ are the weight matrices of rightmost and leftmost weight connections respectively, and similarly $B_{L}$ and $B_{R}$ are the bias vectors of hidden layer neurons and output layer neurons respectively.

\section{B. Use of Error Backpropagation with the Proposed Critic}

Recall the cost measure in (7), which is rewritten below for clarity.

$$
J=\frac{1}{2} \sum_{j=1}^{n} s_{C j}^{2}=\frac{1}{2} \sum_{j=1}^{n}\left(u_{j}-u_{j}^{*}\right)^{2}
$$

where $\underline{u}^{*}$ is the unknown target control signal in some desired sense. According to the EBP based tuning strategy, in order to minimize the cost of (1), if $\sigma$ is defined to be a generic adjustable parameter of the neurocontroller, the adjustment of $\sigma$ is carried out by the rule given as

$$
\dot{\sigma}=-\eta \frac{\partial J}{\partial \sigma}=-\eta \sum_{j=1}^{n} s_{C j} \frac{\partial u_{j}}{\partial \sigma}
$$

where, $\eta$ is the learning rate chosen from the interval $(0,1)$. The rule in (12) is the ordinary backpropagation, and the only modification is the use of new error term denoted by $\underline{s}_{C}$.

\section{Interception Problem and Observations}

In the simulations, missile is kept under an external feedback loop as illustrated in Fig. 3.

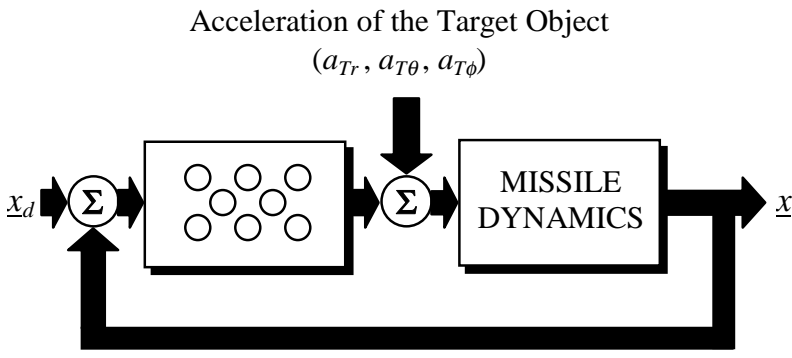

Fig. 3. Structure of the control system

According to the differential equations of the missile, acceleration vector of the target object joins to the feedback 
loop after the controller outputs additively. Apparently, for the interception problem $\underline{x}_{d}=0$ for $\forall t \geq 0$, however, a timevarying $\underline{x}_{d}$ would mean that the controller tries to preserve a relative dynamics between the target and the missile according to what is described by $\underline{x}_{d}$. Therefore, in our simulations we set $\underline{x}_{d}=0$. As the initial conditions, we adopt the data presented in [1], i.e. $r(0)=5 \mathrm{~km}, \theta(0)=-0.2 \mathrm{rad}$, $\phi(0)=0.1 \mathrm{rad}, \dot{r}(0)=-0.51 \mathrm{~km} / \mathrm{hr}, \dot{\theta}(0)=-0.01 \mathrm{rad} / \mathrm{hr}$ and $\dot{\phi}(0)=0.05 \mathrm{rad} / \mathrm{hr}$. The target object has the following acceleration vector entries: $a_{T \theta}=\sin (0.1 t), a_{T \phi}=\cos (0.1 t)$ and $a_{T r}=0$. For the design parameters, we set $\Xi=\mathrm{I}_{3 \times 3}, \delta=0.05$, and $\eta=0.01$. Initally the weights and the biases of the neural network have randomly been set from the interval [0,0.1]. The controller has the configuration 6-6-3. During the simulations, we computed the derivative term in the error measure of (8) by numerical differentiation of $s_{p}$.

An important remark here should emphasize that the dynamic equations of the missile have not been used in any phase of the design procedure. We solely assumed that the dynamics is such that the task is achievable. Furthermore, no initial training has been performed on the controller, which operates on-line.

Under these conditions, we observed the state evolution depicted in Fig. 4. After a fast reaching phase, the missile intercepts the target.
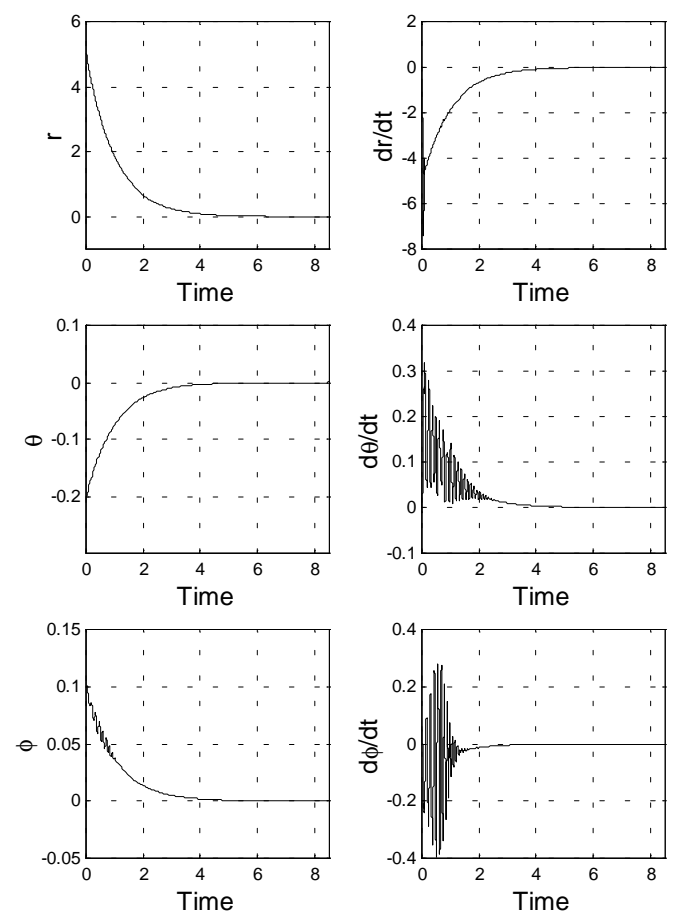

Fig. 4. Behavior of the missile states

In Fig. 5, the acceleration profile of the target and the acceleration input produced by the neurocontroller are illustrated. The effect of hittinggs to the sliding hypersurface is apparent in all three components in the left subplots. The acceleration produced by the controller drives the interception system to zero error state along with the sliding manifold.
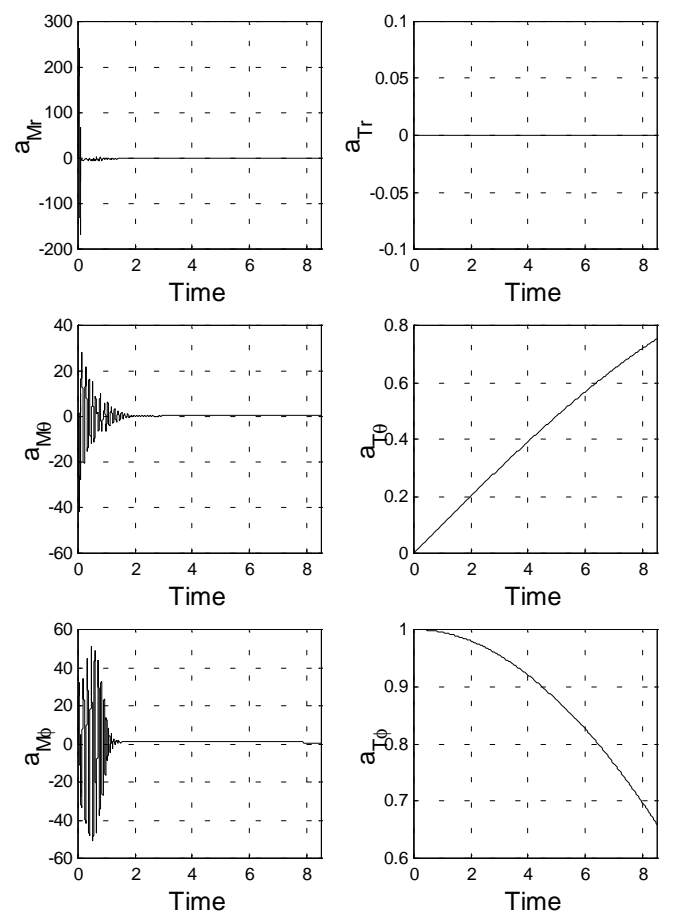

Fig. 5. Acceleration profiles for the missile and the target

In the left subplots of Fig. 6, the behavior in the phase space for each state component are illustrated. The results figure out that the missile quickly enters the sliding mode in the radial direction and several more hittings are observed in the azimuth and pitch angles. The right subplots of the figure depict the time evolution of the adjustable controller parameters, which is denoted by $P$ and defined as given in (13).

$$
P=\sqrt{\Omega_{H}^{T} W_{L}^{T} W_{L} \Omega_{H}+B_{L}^{T} B_{L}+W_{R}^{T} W_{R}+B_{R}^{T} B_{R}}
$$

where, $\Omega_{H}=\left[\begin{array}{llll}1 & 1 & \cdots & 1\end{array}\right]^{T}$ and is of $H \times 1$ dimensional with $H$ being the number of hidden neurons. The quantity above is a good measure of visualizing the evolution in the adjustable parameter space. It should here be stressed that one of the major drawbacks of gradient based approaches is the lack of control over the adjustable parameters. Since the information determining the evolution of the parameter vector is strictly dependent upon the topology of the cost hypersurface, the gradient techniques sometimes compute unnecessarily large parametric displacements. The visualization of the above quantity in this sense will enable us to assess the stability, and therefore application safety, of the proposed scheme. In the top right subplot of Fig. 6, Pmeasure is plotted on linear time axis. It is clear from the 
figure that the parameters receive the excitation during the early phase of the flight, which corresponds to the reaching phase and the suggested error critic of (8) yields nonzero values. When the error vector gets trapped to the sliding manifold, the error prescribed by (8) is very close to zero, and the parameters are not excited at all. The bottom right subplot of Fig. 6 illustrates the $P$-measure in the logarithmic time axis, and the observed behavior justifies the above remarks and the stability in the parameter space.
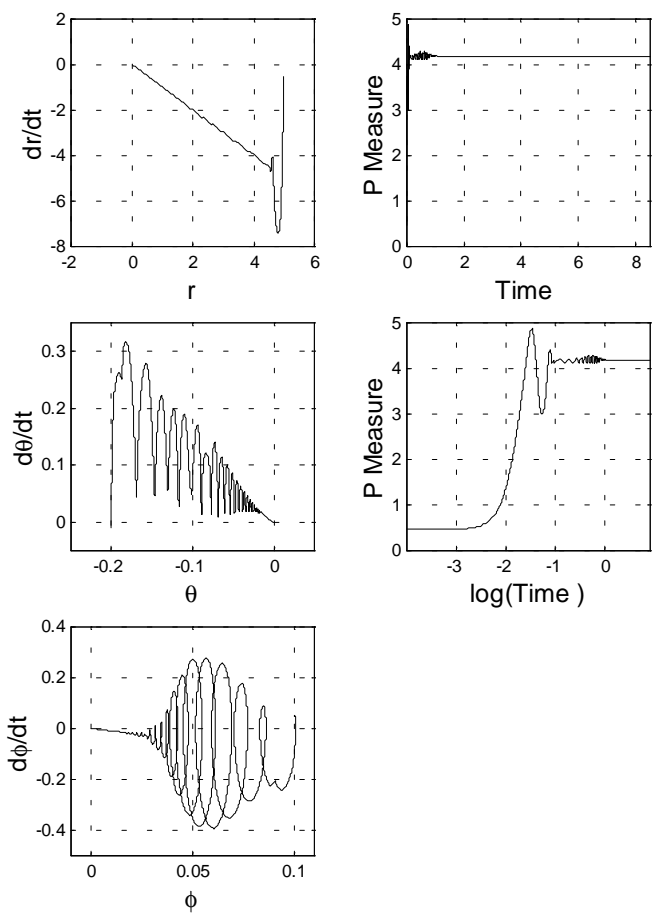

Fig. 6. Behavior of the error components in the corresponding phase planes and the behavior of the parametric cost measure $P$.

\section{CONCLUSIONS}

This paper postulates a novel error measure for backpropagation neural networks. The suggested measure is based on the VSC technique and aims to drive the system into a prespecified sliding mode.

The attractiveness of the approach lies neither solely in the architectural versatility nor only in the algorithmic strength. The insight here should stress the coordination of algorithmic capabilities and architectural degrees of freedom in a collaborative manner. Nor we claim that a missile can be controlled with randomly initialized controller parameters, the conclusion of this simulation study is to explore the performance of a novel scheme on a challenging control problem.

The results observed justify the precise interception claim of the paper with bounded norm evolution in the adjustable parameter space. The approach with the presented observations constitutes a good candidate for tracking control problems.

\section{ACKNOWLEDGMENTS}

This work is supported by NSF (Grant No: 9906233).

\section{REFERENCES}

[1] Liaw, D.-C., Liang, Y.-W. and Cheng, C.-C., "Nonlinear Control for Missile Terminal Guidance," Trans. of the ASME: Journal of Dynamic Systems, Measurement and Control, v.122, pp.663-668, December 2000.

[2]. Jianbo, H. Hongye, S. and Jian. C., "Variable Structure Control for a Class of Fuzzy Dynamics and Its Application to the Design of Autopilot for Missile," Proc. of the $3^{\text {rd }}$ World Congress on Intelligent Control and Automation, June 28-July 2, Hefei, P.R. China, pp.1615$1619,2000$.

[3] Thukral, A. and Innocenti, M., "Variable Structure Autopilot for High Angle of Attack Maneuvers Using On-Off Thrusters," Proc. of the $33^{\text {rd }}$ Conf. on Decision and Control, Lake Buena Vista, FL, pp.3850-3851, 1994.

[4] Han, D. and Balakrishnan, S. N., "Adaptive Critic Based Neural Networks for Control-Constrained Agile Missile Control," Proc. of the American Control Conf., San Diego, CA, pp.2600-2604, June 1999.

[5] Kwan, C., Xu, R., Liu, W., Tan, R. and Haynes, L., "Nonlinear Control of Missile Dynamics," Proc. of the $37^{\text {th }}$ IEEE Conf. on Decision and Control, Tampa, FL, pp.4685-4690, December 1998.

[6] Blumel, A. L., Hughes, E. J. and White, B. A., "Design of Robust Fuzzy Controllers for Aerospace Applications," $18^{\text {th }}$ Int. Conf. of the North American Fuzzy Information Processing Society, New York City, NY, pp.438-442, 1999.

[7] Kim, Y.-S., Han, W.-G. and Kuc, T.-Y., "An Intelligent Missile Autopilot Using Genetic Algorithm," 1997 IEEE Int. Conf. on Computational Cybernetics and Simulation, Orlando, FL, v.2, pp.1954-1959, 1997.

[8] Sira-Ramirez, H. and Colina-Morles, E., "A Sliding Mode Strategy for Adaptive Learning in Adalines", IEEE Transactions on Circuits and Systems - I: Fundamental Theory and Applications, Vol. 42, No.12, pp. 1001-1012, December 1995.

[9] Yu, X., Zhihong, M. and Rahman, S. M. M., "Adaptive Sliding Mode Approach for Learning in a Feedforward Neural Network," Neural Computing \& Applications, Vol. 7, pp. 289-294, 1998.

[10] Yu, X., Efe, M. O. and Kaynak, O., "A Backpropagation Learning Framework for Feedforward Neural Networks," Proc. of the 2001 IEEE Int. Symposium on Circuits and Systems, ISCAS'2001, May 6 9, 2001 Sydney, Australia.

[11] Parma, G. G., Menezes, B. R. and Braga, A. P., "Sliding Mode Algorithm for Training Multilayer Artificial Neural Networks," Electronics Letters, Vol. 34, No. 1, pp. 97-98, January 1998.

[12] Efe, M. O. and Kaynak, O., "A Novel Optimization Procedure for Training of Fuzzy Inference Systems By Combining Variable Structure Systems Technique and Levenberg-Marquardt Algorithm," Fuzzy Sets and Systems, v.122, no.1, pp. 149-161, 2001.

[13] Efe, M. O. and Kaynak, O., "On Stabilization of Gradient Based Training Strategies for Computationally Intelligent Systems," IEEE Transactions on Fuzzy Systems, v.8, no.5, pp.564-575, October 2000.

[14] Efe, M. O., Kaynak, O. and Yu, X., "Sliding Mode Control of a Three Degrees of Freedom Anthropoid Robot by Driving the Controller Parameters to an Equivalent Regime," Trans. of the ASME: Journal of Dynamic Systems, Measurement and Control, v.122, no.4, pp.632-640, December 2000.

[15] Yang, C.-D and Yang, C.-C., "Analytical Solution of Three Dimensional Realistic True Proportional Navigation," Journal of Guidance, Control and Dynamics, v.19, no.3, pp.569-577, 1996. 